\section{A Posteromedial Approach to a Complex Multifragmentary Avulsion Fracture of the Posterior Cruciate Ligament: A Case Report}

Case

While riding a motorcycle, a 37-year-old male injured his knee in a collision with a vehicle. The injury occurred when the patient's motorcycle landed on top of him. The exact location of impact is unknown. The patient complained solely of pain in his right, lower extremity.

Pre-operative clinical examination revealed no neurovascular deficit, but was limited due to significant pain and patient reluctance to participate in range of motion. Radiographs showed an intraarticular fracture of the posterior aspect of the right tibial spine with the dominant fragment displaced approximately $1 \mathrm{~cm}$ posteriorly in the joint space. Computed Tomography (CT) verified a $1 \mathrm{~cm}$ fragment lateral to the posterior tibial plateau (Figure 1). Magnetic Resonance Imaging (MRI) of the knee showed a comminuted fracture involving the tibial plateau as seen on radiographs and $\mathrm{CT}$, an attenuated Anterior Cruciate Ligament (ACL) without a full thickness tear, and an intact Posterior Cruciate Ligament (PCL) attached to the dominant posteriorly displaced fracture fragment (Figure 2). Other ligament pathology included a type I sprain of the fibular collateral ligament (FCL). Examination under anesthesia revealed restricted motion of the knee from 0 to 90 degrees, posterior sag was apparent and posterior drawer test with the knee flexed at 90 degrees showed grade III instability with a soft endpoint. The patient also had a slight increase in dial testing at 30 degrees and varus stress testing at 30 degrees demonstrated a slight laxity with firm endpoint. Based on the patient's age and activity level, operative intervention was deemed appropriate.

\section{Methods}

During the operation, the patient lay in the prone position with
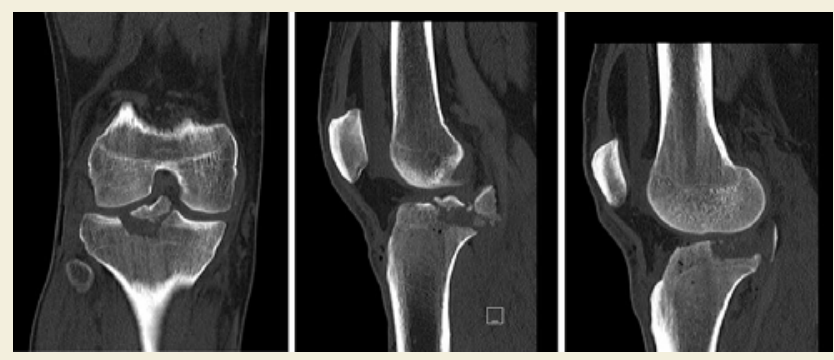

Figure 1: Computed tomography scans showing coronal and sagittal views of the knee.

\section{Journal of \\ Orthopedics \& Rheumatology}

Sophie Strike ${ }^{1}$, Amanda K. Brock ${ }^{3}$, Qais Naziri', Johnathan Bernard ${ }^{1}$ and Bashir Zikria ${ }^{1,2 *}$

${ }^{1}$ Division of Sports Medicine, The Johns Hopkins University, Baltimore, Maryland, United States

${ }^{2}$ Director of Sports Medicine at Johns Hopkins Good Samaritan, Baltimore, Maryland, United States

${ }^{3}$ Towson University, Towson, Maryland, United States

${ }^{4}$ Department of Orthopaedics, State University New York, Brooklyn, New York, United States

*Address for Correspondence

Bashir Zikria, The Johns Hopkins University/Johns Hopkins Bayview Medical Center, 4940 Eastern Ave., \#A665, Baltimore, MD 21224-2780, USA, Tel: 410-550-5400; Fax: 410-550-2899; e-mail: bzikria2@jhmi.edu

\section{Submission: 09 March 2014}

Accepted: 20 May 2014

Published: 27 May 2014

Copyright: @ 2014 Strike S, et al. This is an open access article distributed under the Creative Commons Attribution License, which permits unrestricted use, distribution, and reproduction in any medium, provided the original work is properly cited.

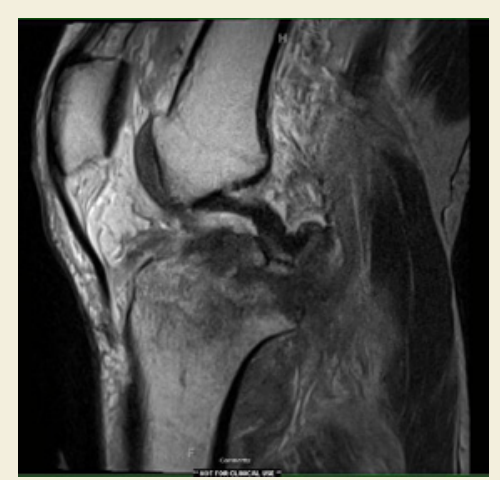

Figure 2: Magnetic resonance imaging showing PCL attached to dominant posteriorly displacement fracture fragment in sagittal plane.

a proximal thigh tourniquet inflated to $200 \mathrm{mmHg}$ on the right leg. An L-shaped incision was marked out with the transverse leg slightly distal to the popliteal crease and longitudinal leg extending five centimeters distally on the medial gastrocnemius (Figure 3). After marking the incision, sharp dissection was carried out and the leg was exsanguinated. Care was taken to protect any branches of the sural nerve. The interval between the semimembranosus and the medial gastrocnemius was bluntly developed proximally and distally. To further expose the posterior capsule of the knee, both the medial gastrocnemius and semimembranosus were retracted to gain exposure. The fracture zone was easily palpable as was the posterior aspect of the knee joint. The capsule was well visualized using appropriate retraction, the inferior medial geniculate artery was identified, dissected free, and then cauterized thoroughly. Capsulotomy was then performed proximally and distally taking care to protect all structures with proper retraction. The joint space was accessed and several comminuted fracture fragments were irrigated 
out. The menisci were visualized and the PCL was found to be attached to a 1.5 by 1.1 centimeter fragment of bone. Additionally, there was a large intra-articular fragment measuring approximately 2.5 by three centimeters in dimension. This appeared to compromise a significant portion of the posterior lateral tibial plateau. However, the posterolateral corned appeared intact. Following cleaning of loose debris, the fracture bed was irrigated copiously. The intra-articular fragment was keyed into position to anatomic reduction. Once the intra-articular fragment was well placed, two diverging K-wires and a cannulated screw held it in position. The PCL avulsion fragment with the attached ligament was then reduced down to the most posterior aspect of the plateau, and was also held in place with a K-wire to attain anatomical reduction of the fragment. After taking fluoroscopic imaging to confirm the alignment of the reduction (Figure 4), holes were drilled to place two partially threaded cannulated lag scews into the intra-articular fragment; one of which was placed with a washer, due to bone quality and to provide a buttress, and the other without a washer. Once achieving a tight fit, a third partially threaded cannulated lag screw with a washer was used to secure the avulsion fracture fragment with the PCL attached. All the screws were adequately tightened to achieve excellent compression across the fracture plane and screw trajectory was perpendicular to the fracture lines. With confirmation of anatomic reduction through fluoroscopic imaging (Figure 5), the K-wires were removed and joint spaces were irrigated copiously. The tourniquet was let down with a total time of 115 minutes. Subcutaneous tissue and skin were closed appropriately and the patient was placed in a knee immobilizer with bump for anterior drawer.

Postoperatively the patient was remained non-weight bearing on the right lower extremity in a knee immobilizer with the anterior drawer bump at all times. Mobility on axillary crutches with a swing through pattern was permitted five days postoperative. The patient was locked in extension for three weeks to allow stabilization of fracture and to allow ligamentous healing. However the brace was opened for physical therapy which was started on POD\#2.The patient was permitted to begin passive range of motion of the knee, straight leg raises, quad sets, and ankle range of motion in physical therapy. Partial weight bearing on the lower extremity was initiated after eight weeks and progressed to full weight bearing as tolerated by the

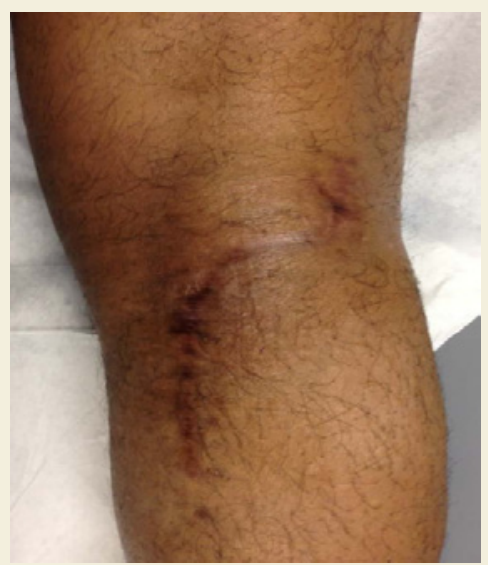

Figure 3: Patient's leg showing scar from L-shaped incision.
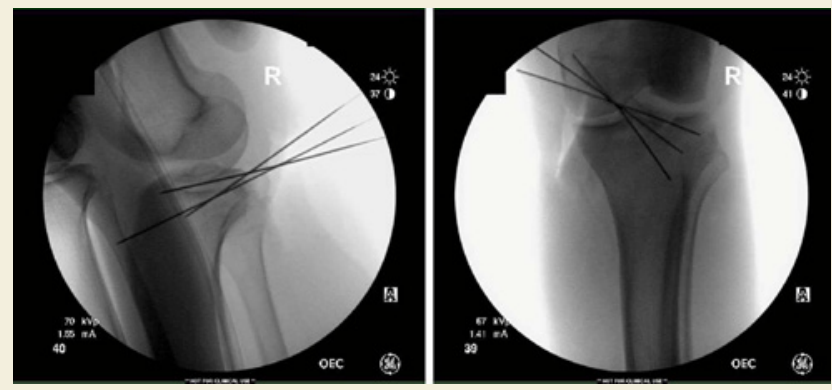

Figure 4: Fluoroscopic imaging showing placed K-wires.
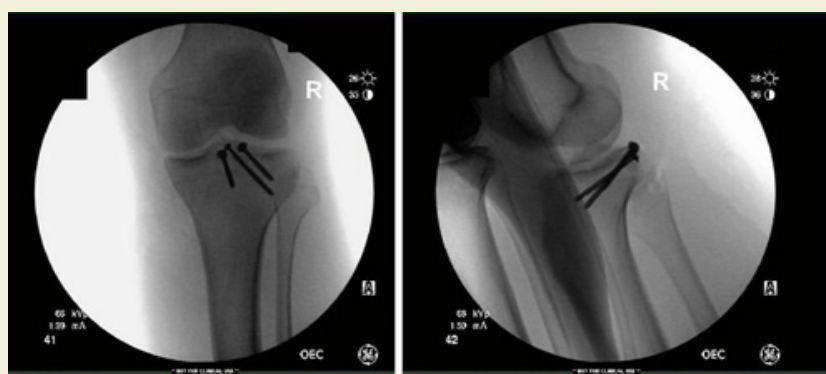

Figure 5: Fluoroscopic imaging showing anatomical reduction with screws.

patient. At the 14 week postoperative follow-up examination, the patient's knee had regained range of motion from zero to 125 degrees (Figure 6), a posterior drawer test revealed grade I laxity with an excellent endpoint, and he had no posterior sag. Radiographs taken at the 14 week postoperative appointment showed healed fractures with excellent fracture alignment and implant position (Figure 7).

\section{Discussion}

The origin of the PCL is located on the lateral border of the medial femoral condyle and the insertion falls on the posterior intercondylar fossa $1.0 \mathrm{~cm}$ below the joint line on the tibia. It has two bundles, anterolateral and posteromedial bundles, that help the PCL act as a primary restraint to posterior tibial translation and a secondary restraint to varus, valgus, and external rotation forces [1]. Individually, these bundles serve further functional roles in the dynamic knee. Specifically, the anterolateral bundle acts as a restraint to posterior tibial translation at mid-range knee flexion while the posteriomedial bundle acts as a restraint to posterior tibial translation during extension and hyperflexion [2].

The epidemiology of PCL injuries is poorly understood. Given the subtlety of physical exam and radiographic findings, the diagnosis is often missed. Several authors have attempted to better characterize these injuries to help clinicians in more accurate diagnosis. Schulz and associates retrospectively reviewed the demographic, mechanism, radiographic, and physical exam findings of 494 patients with confirmed acute and chronic PCL deficient knees. The mean age at the time of injury was $27.5+/-9.9$ years with traffic accidents and athletic injuries accounting for the majority of the causes, $45 \%$ and $40 \%$ respectively [3]. The most common mechanisms were dashboard injuries and falls onto a flexed knee with a plantar flexed foot [3]. 


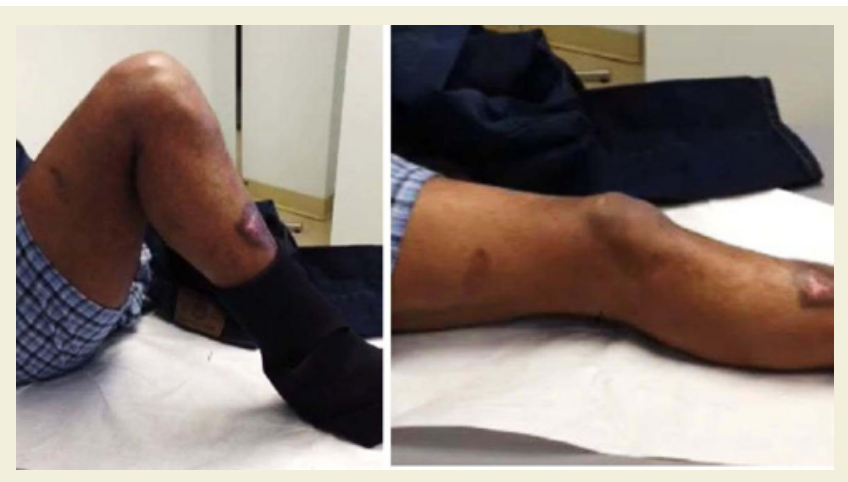

Figure 6: Images taken during 14 week follow up appointment showing knee range of motion.
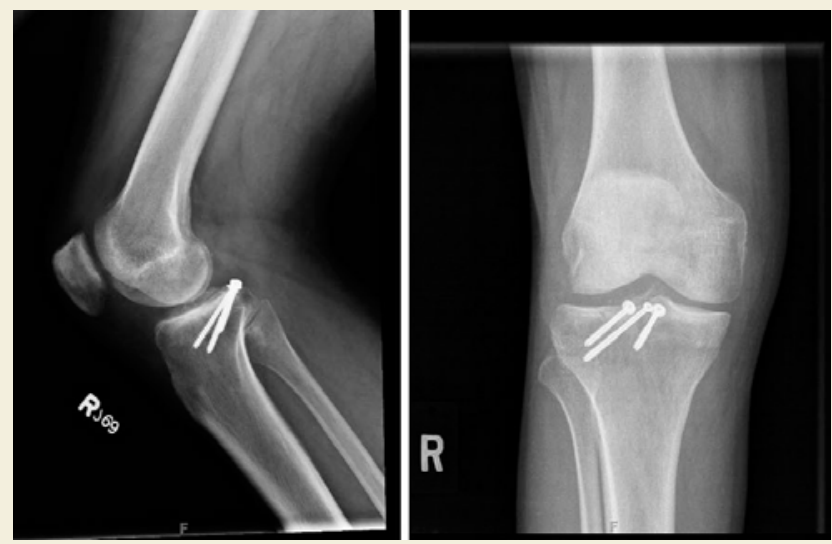

Figure 7: Radiographs of knee showing anatomic alignment of healed fractures.

PCL injuries constitute a spectrum of PCL insufficiency, ranging from frank PCL rupture of the ligament with a tear at either its origin or insertion, or sprain with residual laxity. It may also include PCL insufficient knees with combined posterior lateral corner injuries or multi-ligamentous knee injuries (including any combination of anterior cruciate ligament, medical collateral ligament, or lateral collateral ligament). Few case reports have described avulsion fractures of the PCL $[4,5]$. Isolated PCL injuries, including tibial avulsion fractures at the site of the PCL insertion, are not common and thus are not extensively described nor are the biomechanics of the inury well-understood [6].

We describe an even more rare PCL avulsion fracture with an associated lateral condyle fracture fragment. Many PCL avulsion fractures have been described; however, many of these injuries are solely PCL avulsion fractures amenable to arthroscopic reduction and fixation [7-15]. However, to the best of our knowledge there is only two reports in the literature of a similar associated fracture patterns [16]. Muhm et al describe using a posteriocentral approach for the treatment of shearing posterior tibial plateau fractures and PCL avulsion fractures[17]. In their review of 33 patients with such injurie treated with approach, they reported few superficial nerve complications, two posterior superficial infections, and no pathological scar tissue [17]. Ogawa et al describe a PCL avulsion-mediated fracture of the lateral tibial condyle with minimal displacement while the fracture fragments in our patient were significantly displaced within the posterior knee joint leaving a large defect in the posterior aspect of the lateral tibial plateau. We believe this more significant injury has not yet been described. Unfortunately, the mechanism of injury is unknown in this case as the patient does not recall the details of his leg position during the injury. As noted by Ogawa et al a posteriorly-directed force on the tibia combined with a significant distraction force may have contributed to the large displacement of the fracture fragments and the lack of posterior capsule injury that would be expected from a pure hyperextension injury [16].

Primary repair is accepted for surgical management of tibial avulsion fractures [18]. The notable fracture displacement required extensive open exposure. A previously-described L-shaped approach to the posteromedial knee was used [19]. Dissection medial to the medial head of the gastrocnemius with development of the plane between the medial head and the semimembranosus and subsequent extensive lateral retraction of the gastrocnemius was used to gain access to the posterior capsule, as described. The extensive involvement of the lateral tibial condyle, however, necessitated improved lateral exposure which was achieved with additional lateral retraction of the medial head of the gastrocnemius, cauterization of the inferior geniculate artery and careful palpation of the popliteal artery at regular intervals to ensure its integrity throughout the procedure.

Having sustained a relatively severe injury with a two-fragment fracture pattern rarely described and notable compromise of the integrity of the lateral tibial condyle, our patient was prepared for a less-than satisfactory outcome. Fracture alignment and fixation achieved intra-operatively undoubtedly contributed to near-anatomic fracture healing as noted in radiographs at 14 weeks post-operative. This healing and compliance with post-operative weight-bearing and range of motion instructions allowed our patient to attain 125 degrees of knee flexion by his three-and-a-half months post-operative examination.

\section{References}

1. Tajima G, Nozaki M, Iriuchishima T, Ingham SJ, Shen W, et al. (2009) Morphology of the tibial insertion of the posterior cruciate ligament. J Bone Joint Surg Am 91: 859-866.

2. Race A, Amis AA. (1994) The mechanical properties of the two bundles of the human posterior cruciate ligament. Journal of Biomechanics 27: 13-24.

3. Schulz MS, Russe K, Weiler A, Eichhorn HJ, Strobel MJ (2003) Epidemiology of posterior cruciate ligament injuries. Arch Orthop Trauma Surg 123: 186191.

4. Meyers MH (1975) Isolated avulsion of the tibial attachment of the posterior cruciate ligament of the knee. J Bone Joint Surg Am 57: 669-672.

5. Ross AC, Chesterman PJ (1986) Isolated avulsion of the tibial attachment of the posterior cruciate ligament in childhood. J Bone Joint Surg Br 68: 747.

6. McAllister DR, Petrigliano FA (2007) Diagnosis and treatment of posterior cruciate ligament injuries. Curr Sports Med Rep 6: 293-299.

7. Bonin N, Jeunet L, Obert L, Dejour D (2007) Adult tibial eminence fracture fixation: arthroscopic procedure using K-wire folded fixation. Knee Surg Sports Traumatol Arthrosc 15: 857-862.

8. Deehan DJ, Pinczewski LA (2001) Arthrosopic reattachment of an avulsion fracture of the tibial insertion of the posterior cruciate ligament. Arthroscopy 
Citation: Strike S, Brock AK, Naziri Q, Bernard J, Zikria B. A Posteromedial Approach to a Complex Multifragmentary Avulsion Fracture of the Posterior Cruciate Ligament: A Case Report. J Orthopedics Rheumatol. 2014;1(3): 4.

17: 422-425.

9. Espejo-Baena A, Lopez-Arevalo R, Urbano V, Montanez E, Martın F (2000) Arthoscopic repair of the posterior cruciate ligament: two techniques. Arthroscopy 16: 656-660

10. Horas U, Meissner SA, Heiss C, Schnettler R (2010) Arthoscopic fixation of posterior cruciate ligament avulsion fractures: a new minimally invasive technique. Knee Surg Sports Traumatol Arthrosc 18: 781-783.

11. Littlejohn SG, Geissler WB (1995) Arthroscopic repair of a posterior cruciate ligament avulsion. Arthroscopy 11: 235-238.

12. Meyers MH (1975) Isolated avulsion fractures of the tibial attachment of the posterior cruciate ligament of the knee. J Bone Joint Surg. 57: 669-672.

13. Shino K, Nakata K, Mae T, Yamada Y, ShiozakiY, Toritsuka Y (2003) Artroscopic fixation of tibial bony avulsion of the posterior cruciate ligament. Arthroscopy 19: E12.
14. Torisu T (1977) Isolated avulsion fractures of the tibial attachment of the posterior cruciate ligament. J Bone Joint Surg 59: 68-72.

15. Veselko M, Saciri V (2003) Posterior approach for arthroscopic reduction and antegrade fixation of avulsion fracture of the posterior cruciate ligament from the tibia with cannulated screw and washer. Arthroscopy 19: 916-921.

16. Ogawa H, Sumi H, Shimizu K (2010) Posterior cruciate ligament mediated avulsion fracture of the lateral tibial condyle: a case report. J OrthopSurg Res 5: 67.

17. Muhm M, Schneider P, Ruffing T, Winkler H (2013) Posterocentral approach to the posterior tibial plateau. Reconstruction of tibial plateau fractures and avulsions of the posterior cruciate ligament. Unfallchir.

18. Chen $\mathrm{CH}$ (2007) Surgical treatment of posterior cruciate ligament injury Chang Gung Med J 30: 480-492.

19. Zhang CL, Xu H, Li MQ (2006) Posteromedial approach of gastrocnemius for reduction and internal fixation of avulsed tibial attachment of posterior cruciate ligament. Chin J Traumatol 9: 25-28. 